\title{
Comparative Study of Phytochemical Profiles and Morphological Properties of Some Damask Rose From Iran
}

\author{
University of Tehran \\ Azizollah Khandan-Mirkohi \\ University of Tehran \\ Mohsen Kafi \\ University of Tehran \\ Zabihollah Zamani \\ University of Tehran \\ Omid Rasouli \\ Tarbiat Modares University \\ Arezoo Shaghaghi \\ University of Urmia \\ Mahnaz Kiani \\ Texas A\&M University
}

Mohammad Omidi ( $\sim$ m.omidighale@ut.ac.ir)

\section{Research Article}

Keywords: Damask rose, Essential oil, GC-mass spectrometry, Perfume, Volatile compounds

Posted Date: March 7th, 2022

DOI: https://doi.org/10.21203/rs.3.rs-1401571/v1

License: (c) (i) This work is licensed under a Creative Commons Attribution 4.0 International License. Read Full License 


\section{Abstract}

Background: Rosa damascena is an aromatic rose species, is cultivated for its essential oil, and has wide application in perfume, cosmetic, pharmaceutical, and food industries in the world. This experiment was conducted to evaluate essential oil and morphological variations of $26 \mathrm{Damask}$ rose genotypes. For this purpose, the effect of harvest time i.e. early morning or evening, and sampling type i.e. fresh or dried petals was evaluated on oil content. In addition, the composition of essential oil of the genotypes was determined using Gas chromatography-mass spectrometry (GC-MS).

Results: Results showed that early morning was the preferable time for flower collection based on oil content quantification. Furthermore, the oil yield of fresh petals was higher than the dried petals. Twenty-five volatile compounds were found in the extracted oils. $\beta$-damascenone, a key marker for the quality of rose oil, was found in 22 genotypes and was more than 1.5\% concentration in G3, G6, and G11 genotypes. The highest components of the oil in Damask rose genotypes were nonadecane (42.51\%), $\beta$-citronellol (40.82\%), $n$-heneicosane (34.69\%), geraniol (27.76\%), and n-tricosane (14.2\%). A wide variation in flower characteristics, such as petal color (from white to nearly red) and petal numbers from about 25 to 95, were also recorded. The G2, G5, and G15 genotypes originated from Isfahan, Fars, and Kerman respectively were selected based on petal number, flower weight, and essential oil content in fresh and dried petals.

Conclusions: Results suggest that the morphological and biochemical diversity of Damask rose genotypes can be used effectively to characterize genetic diversity between different genotypes and to select special traits in the breeding program

\section{Background}

Damask rose (Rosa damascena Mill.) is one of the supreme fragrance species in the Rosaceae family that is derived from Rosa gallica and Rosa moschata [1]. This species is cultivated for its ornamental value and also for essential oil extraction in most parts of the northern hemisphere [2,3]. Iran has been introduced as the genetic diversity center and the origin of Damask roses [4-10]. Nowadays, this species is cultivated extensively in Bulgaria, Iran, Turkey, France, Italy, Morocco, the USA, and India [11]. The global production of rose oil is about 4.5 tones per year [12]. The global rose oil market was valued 278.7 million USD in 2018 [13]. Products of Damask rose, including essential oil, rose water, rose concrete, dried petals, dried flower buds, and rose absolute, are used in perfume, cosmetic, pharmaceutical, and food industries. Several pharmacological attributes, such as antibacterial, antioxidant, and anti-HIV effects have been found in rose oil [14-16].

Rosa damascena is cultivated in widespread ecological conditions, but specific climatic conditions are needed to produce high-quality essential oil. The quality of essential oil and flower yield of $R$. damascena is mainly affected by geographical origin and climate conditions, time and stage of flower harvesting, method of extraction [5], and agriculture practices [17-21]. Rosa damascena grows wild in some parts of Iran and hasbeen propagated vegetatively and cultivated for a long time [6]. Thus, various cultivars of Damask rose have been selected during the long cultivation history, and it has also been crossed naturally with local species [22]. The phenotypic homogeneity caused by continuous vegetative reproduction and environmental effects makes it possible for mass production of this crop to produce rose oil $[5,9,23]$.

The most important compounds of rose oil are $\beta$-citronellol, nonadecane, geraniol, eugenol, heneicosane, and phenols such as eugenol [24, 25]. In addition, some factors such as the concentration of ethanol used for extraction, storage period, and production conditions of flowers, also would affect the amounts of key compounds in rose oil [5]. Several studies have been conducted on the chemical composition of essential oil in various populations of Damask rose by GC/MS through different extraction methods [3, 9, 15, 26-28], and also on genetic and morphological diversity of Rosa damascena [6, 10, 29-32]. The effect of micro-climate on Damask rose cultivation and the oil composition has been also reported [32, 33].

As we have access to the wide genetic diversity of Rosa damascena in Iran, it will be valuable to characterize their specific morphology and biochemical characters in more detail. Thus, the present study was carried out to determine the variations in the flower yield and morphological and chemical compositions of 26 different Iranian Damask rose genotypes by using gas chromatography-mass spectrometry (GC-MS).

\section{Materials And Methods}

\section{Plant materials and collection site}

Damask rose genotypes that were selected for this study consisted of 26 genotypes These genotypes were previously collected from several parts of Iran (Table 1) and established in the research station of the Horticultural sciences department, the University of Tehran, Karaj, Iran (latitude $35^{\circ} .77^{\prime} \mathrm{N}$, longitude $50^{\circ} .93^{\prime} \mathrm{E}$ and altitude $1251 \mathrm{~m}$ ), based on a randomized block design with three replications in $2004{ }^{[8]}$. All samples were the same because of their yearly pruning. The average plant high was $165 \mathrm{~cm}$ and plant diameter was $123 \mathrm{~cm}$. The current experiment was carried out during $2016-2018$. 
Table 1

Damask rose genotypes assessed in this study, their province of origin, collection site, and petal color

\begin{tabular}{|c|c|c|c|c|c|c|}
\hline Genotype No. & Province of origin & Collecting site & Longitude $\left(^{\circ}\right)$ & Latitude $\left({ }^{\circ}\right)$ & Altitude & Petal color \\
\hline G1 & East Azerbaijan & Kashan, collection of Taghtiran Company & 51.05 & 34.02 & 1814 & Pink \\
\hline G2 & Isfahan & Kashan, collection of Taghtiran Company & 51.05 & 34.02 & 1814 & Dark pink \\
\hline G3 & Tehran & Kashan, collection of Taghtiran Company & 51.05 & 34.02 & 1814 & Pink \\
\hline G4 & Isfahan & Kashan, collection of Taghtiran Company & 51.05 & 34.02 & 1814 & Dark pink \\
\hline G5 & Fars & Kashan, collection of Taghtiran Company & 51.05 & 34.02 & 1814 & White \\
\hline G6 & Fars & Darab, Lyzangan & 54.98 & 28.66 & 2018 & Pale pink \\
\hline G7 & Fars & Darab, Rostagh & 55.06 & 28.44 & 1314 & Pink \\
\hline G8 & Fars & Darab, Ghale Biaban & 54.87 & 28.52 & 1339 & Pink \\
\hline G9 & Fars & Darab, Lyzangan & 54.99 & 28.67 & 2070 & Pale pink \\
\hline G10 & Fars & Maimand, Sahra sefid & 52.79 & 28.83 & 1480 & White \\
\hline G11 & Fars & Maimand, Kang & 52.83 & 28.87 & 1649 & Pink \\
\hline G12 & Fars & Maimand & 52.76 & 28.86 & 1548 & Pink \\
\hline G13 & Kerman & Bardsir & 56.58 & 29.90 & 2070 & Pale pink \\
\hline G14 & Kerman & Bardsir & 56.61 & 29.87 & 2095 & Pink \\
\hline G15 & Kerman & Mahan & 57.24 & 30.12 & 1823 & Pink \\
\hline G16 & East Azerbaijan & Osco & 46.13 & 37.92 & 1567 & Pink \\
\hline G17 & East Azerbaijan & Tabriz & 46.43 & 38.01 & 1673 & Pink \\
\hline G18 & East Azerbaijan & Osco & 46.11 & 37.89 & 1575 & Pink \\
\hline G19 & East Azerbaijan & Osco & 46.18 & 37.90 & 1685 & Dark pink \\
\hline G20 & East Azerbaijan & Ahar & 47.04 & 38.44 & 1387 & Pink \\
\hline G21 & Isfahan & Kashan & 51.47 & 33.94 & 979 & Pink \\
\hline $\mathrm{G} 22$ & Isfahan & Kashan & 51.53 & 33.94 & 974 & Pink \\
\hline $\mathrm{G} 23$ & Isfahan & Kashan & 51.61 & 33.93 & 946 & Pale pink \\
\hline G24 & Razavi Khorasan & Mashhad & 59.46 & 36.61 & 1122 & Pink \\
\hline G25 & Razavi Khorasan & Mashhad & 59.43 & 36.38 & 1092 & Pink \\
\hline G26 & East Azerbaijan & Tabriz & 46.40 & 37.98 & 1783 & White \\
\hline
\end{tabular}

\section{Evaluation of plant vegetative and flower characteristics}

The morphological characters, such as plant height, crown diameter, number of main stems in each plant, number of flowers in main stems, angle of the secondary branches, internode length, and thorn density (one to five from high to low), were determined in twelve years old plants. Additionally, the length of stipule, peduncle, receptacle, and flower bud's length and diameter prior to the opening stage were measured. Petal colors were determined visually and also measured with a colorimeter (Minolta CR-400 Chroma meter, Konica Minolta Sensing, Inc., Osaka, Japan) using the following parameters: L* (lightness), a* (redness), and $b^{\star}$ (yellowness). Color parameters were obtained through reflectance values and Chroma calculated by the following formula [34].

Chroma $=\sqrt{a^{2}+b^{2}}$

\section{Isolation and content of essential oil}

For the extraction of essential oil, fresh flowers from each accession were randomly collected (20 fresh flowers for each accession). Flowers were harvested both in the morning and evening. For dried samples, the collected flowers were spread on wire shelves and kept in the shade for two weeks at room temperature [35]. A total of $200 \mathrm{~g}$ of fresh petals and $55 \mathrm{~g}$ of dry petals (equivalent to $200 \mathrm{~g}$ fresh petals) subjected to hydrodistillation using $400 \mathrm{ml}$ distilled water in a Clevenger for 3 hours with three replications [64]. The essential oil was measured directly in the extraction burette and the oil content ( $\mathrm{V} / \mathrm{w}$ ) in flower was expressed as percentage on a fresh weight basis of essential oil per $200 \mathrm{~g}$ of fresh petals. The extracted oils were transferred into vials and stored at $4{ }^{\circ} \mathrm{C}$ in the dark until used.

Gas chromatography (GC-FID) and (GC-MS) 
GC-MS analysis of the oil samples was performed on a Thermo-UFM (Ultra-Fast model, Italy) gas chromatograph equipped with a P5 (non-polar) capillary column $(10 \mathrm{~m} \times 0.1 \mathrm{~mm})$, which employed helium $(0.5 \mathrm{ml} / \mathrm{min})$ as the carrier gas to split injection at $1: 100$. The oven temperature was set at $60^{\circ} \mathrm{C}$ for $30 \mathrm{~min}$, FID detector temperature was programmed at $285^{\circ} \mathrm{C}$ at the rate of $80^{\circ} \mathrm{C} / \mathrm{min}$ and injector temperature was $280^{\circ} \mathrm{C}$. The relative amounts of individual components were calculated based on the $\mathrm{GC}$ peak areas by using a normalization method regarding response factor. The essential oil constituents were identified following an injection of n-alkanes $\left(\mathrm{C}_{8}-\mathrm{C}_{24}\right)$ under the same conditions and confirmed according to Wiley 275-L library and literature [36-38]. The compounds were identified using commercial mass spectral libraries (NIST 05, Wiley 7th Mass spectra register) [37].

\section{Statistical analysis}

For the evaluation of morphological characteristics of vegetative and flower parts, the experiment was arranged in a randomized complete block design (RCBD) with three replications. Mean values were compared at $95 \%(p \leq 0.05)$ and $99 \%(p \leq 0.01)$ confidence intervals using the LSD test by Minitab ver.16. [39].

\section{Results}

\section{Oil content in fresh and dry petals}

In the majority of selected Damask rose genotypes, petals harvested in the morning time for dried and fresh petals had higher oil content in comparison with samples collected in the evening time with the exception of G9, G11and G23 genotypes that opposite trend was found (Fig. 1).The highest oil content in fresh petals was found in G21 for morning and evening harvest time 0.14 and 0.15 (v/w\%) Respectively. Additionally, in G18, G19, G20, G24, and G25 genotypes the total volume of essential oil content in both harvesting times was similar (Fig. 1). In dried petals, the time of harvesting also affected the oil content. However, the oil content in dried petals generally was lower than the fresh petals. Although in most of the genotypes, the higher oil content was recorded in the morning time harvested dried petals, three genotypes (G1, G2, and G4) had the same content of essential oil in both dried morning and evening times harvested petals. On the other hand, due to later flower blooming in G22 (0.05 v/w\%) and G24 (0.04 v/w\%) genotypes, the oil content in dried petals harvested in the evening time was more than the morning time collected samples (Fig. 2).

\section{Morphological traits}

There were clear differences in morphological characteristics between selected Damask rose genotypes (Table 2). The correlation matrix among morphological traits of $R$. damascena showed that the plant height was significantly $(P=0.01)$ and positively correlated with the branch angle $(r=0.21)$, number of stems per plant $(r=0.57)$, flower bud length $(r=0.46)$ and flower peduncle length $(r=0.45)$ (Table 3$)$. Thorn density was positively correlated with the number of petals $(P=0.01)$. Moreover, a negative correlation $(r=-0.39)$ was found between thorn density and branch angle. A significant $(P=0.01)$ negative $(r=-0.56)$ correlation was found between flower peduncle length and thorn density. Thorn density had a negative relation with all traits, except with the bud diameter (Table 3). The flower peduncle length was positively correlated with most traits evaluated in this study. Different petal colors, from white to dark purple, were observed in the selected Damask rose genotypes (Table 1). The measurements of color parameters gave different values of $L^{*}, a^{*}$, and $b^{\star}$. The results showed highly significant differences among genotypes for all color traits (Table 4). Chroma values also were different between genotypes. 
Table 2

Measured morphological characters (mean $\pm \mathrm{SE}$ ) of 26 Damask rose genotypes

\begin{tabular}{|c|c|c|c|c|c|c|c|c|c|c|c|}
\hline $\begin{array}{l}\text { Genotype } \\
\text { No }\end{array}$ & $\begin{array}{l}\text { Plant } \\
\text { height } \\
\text { (cm) }\end{array}$ & $\begin{array}{l}\text { Plant crown } \\
\text { diameter(cm) }\end{array}$ & $\begin{array}{l}\text { No of } \\
\text { main } \\
\text { stems } \\
\text { per plant }\end{array}$ & $\begin{array}{l}\text { No of } \\
\text { nodes in } \\
\text { the } \\
\text { branch }\end{array}$ & $\begin{array}{l}\text { Internode } \\
\text { length } \\
(\mathrm{mm})\end{array}$ & $\begin{array}{l}\text { Secondary } \\
\text { branches } \\
\text { angle }\end{array}$ & $\begin{array}{l}\text { Thorns } \\
\text { density }\end{array}$ & $\begin{array}{l}\text { Leaf } \\
\text { stipule } \\
\text { length } \\
(\mathrm{mm})\end{array}$ & $\begin{array}{l}\text { Leaf } \\
\text { stipule } \\
\text { width } \\
(\mathrm{mm})\end{array}$ & $\begin{array}{l}\text { Peduncle } \\
\text { length } \\
(\mathrm{mm})\end{array}$ & $\begin{array}{l}\text { Receptacle } \\
\text { length } \\
(\mathrm{mm})\end{array}$ \\
\hline G1 & $111.8+5.5$ & $69.4 \pm 5.13$ & $14.2 \pm 2.0$ & $10.5 \pm 1.7$ & $23.9 \pm 1.2$ & $73.1 \pm 5.5$ & $4.0 \pm 00$ & $15.9 \pm 1.0$ & $6.9 \pm 0.4$ & $14.8 \pm 1.1$ & $10.2 \pm 1.3$ \\
\hline G2 & $160.5+4.7$ & $123.9 \pm 8.7$ & $19.0 \pm 1.8$ & $27.2 \pm 3.3$ & $34.0 \pm 3.9$ & $83.3 \pm 1.6$ & $4.0 \pm 00$ & $16.8 \pm 1.4$ & $20.4 \pm 1.9$ & $22.9 \pm 1.8$ & $7.7 \pm 0.3$ \\
\hline G3 & $111.2+35$ & $104.0 \pm 3.7$ & $14.2 \pm 0.8$ & $19.5 \pm 1.7$ & $29.3 \pm 2.7$ & $81.1 \pm 2.3$ & $4.0 \pm 00$ & $19.0 \pm 1.2$ & $8.4 \pm 0.8$ & $18.8 \pm 1.2$ & $14.3 \pm 1.0$ \\
\hline G4 & $185.2+3.7$ & $139.2 \pm 4.2$ & $16.4 \pm 0.9$ & $34.1 \pm 3.2$ & $32.8 \pm 3.1$ & $85.5 \pm 1.3$ & $2.0 \pm 00$ & $18.5 \pm 0.7$ & $10.1 \pm 0.6$ & $26.7 \pm 1.5$ & $10.6 \pm 0.4$ \\
\hline G5 & $129.4+4.5$ & $107.8 \pm 4.8$ & $16.9 \pm 1.0$ & $23.8 \pm 1.4$ & $29.0 \pm 2.4$ & $66.7 \pm 1.6$ & $5.0 \pm 00$ & $20.0 \pm 1.0$ & $15.3 \pm 0.9$ & $22.2 \pm 2.0$ & $6.4 \pm 0.2$ \\
\hline G6 & $174.1+6.1$ & 152. $9 \pm 5.6$ & $18.3 \pm 1.7$ & $27.2 \pm 2.0$ & $27.0 \pm 2.3$ & $78.3 \pm 2.8$ & $3.1 \pm 0.2$ & $14.6 \pm 1.0$ & $7.6 \pm 0.5$ & $28.9 \pm 2.5$ & $8.9 \pm 0.4$ \\
\hline G7 & $177.1+2.8$ & 135. $8 \pm 2.3$ & $17.2 \pm 1.1$ & $32.1 \pm 5.7$ & $36.5 \pm 4.0$ & $83.3 \pm 1.6$ & $1.0 \pm 00$ & $26.6 \pm 1.9$ & $8.0 \pm 0.5$ & $32.2 \pm 2.7$ & $10.6 \pm 0.4$ \\
\hline G8 & $194.4+3.3$ & $148.0 \pm 3.3$ & $15.3 \pm 0.6$ & $46.4 \pm 4.1$ & $37.2 \pm 2.6$ & $86.1 \pm 1.4$ & $1.0 \pm 00$ & $25.2 \pm 1.1$ & $10.6 \pm 0.8$ & $29.5 \pm 1.8$ & $10.8 \pm 0.2$ \\
\hline G9 & $168.3+8.9$ & $133.5 \pm 4.3$ & $15.0 \pm 0.7$ & $41.9 \pm 6.4$ & $36.3 \pm 2.6$ & $79.4 \pm 2.4$ & $5.0 \pm 00$ & $26.2 \pm 1.7$ & $11.4 \pm 0.7$ & $24.1 \pm 0.9$ & $10.2 \pm 0.7$ \\
\hline G10 & $200.6+4.8$ & $113.0 \pm 3.6$ & $22.2 \pm 1.5$ & $35.9 \pm 2.6$ & $32.8 \pm 3.2$ & $82.2 \pm 2.2$ & $2.0 \pm 00$ & $22.0 \pm 1.2$ & $9.4 \pm 0.9$ & $30.0 \pm 1.7$ & $9.8 \pm 0.4$ \\
\hline G11 & $116.5+2.3$ & $104.0 \pm 4.8$ & $11.4 \pm 0.9$ & $16.9 \pm 2.4$ & $33.2 \pm 3.1$ & $86.6 \pm 1.1$ & $1.0 \pm 00$ & $24.1 \pm 1.2$ & $10.6 \pm 0.4$ & $29.7 \pm 1.5$ & $10.8 \pm 0.2$ \\
\hline G12 & $205.4+3.0$ & 153. $9 \pm 4.4$ & $22.1 \pm 1.3$ & $38.2 \pm 4.5$ & $35.0 \pm 1.7$ & $78.3 \pm 2.0$ & $1.0 \pm 00$ & $17.0 \pm 0.7$ & $8.1 \pm 0.4$ & $32.6 \pm 1.7$ & $10.1 \pm 0.5$ \\
\hline G13 & $213.8+5.5$ & $150.0 \pm 2.8$ & $35.3 \pm 1.1$ & $32.0 \pm 2.1$ & $33.7 \pm 3.6$ & $75.0 \pm 2.0$ & $2.3 \pm 0.1$ & $19.5 \pm 0.4$ & $7.5 \pm 0.3$ & $29.5 \pm 2.6$ & $9.4 \pm 0.4$ \\
\hline G14 & $182.5+3.5$ & $114.0 \pm 3.1$ & $19.8 \pm 1.4$ & $31.4 \pm 2.8$ & $30.2 \pm 2.4$ & $80.0 \pm 2.3$ & $1.0 \pm 00$ & $20.5 \pm 1.6$ & $9.4 \pm 0.7$ & $30.2 \pm 1.9$ & $9.2 \pm 0.4$ \\
\hline G15 & $201.3+4.3$ & $160.0 \pm 3.3$ & $28.1 \pm 0.9$ & $33.0 \pm 2.0$ & $28.1 \pm 1.9$ & $77.8 \pm 2.6$ & $2.3 \pm 0.1$ & $21.5 \pm 1.2$ & $7.5 \pm 0.8$ & $35.7 \pm 2.0$ & $9.2 \pm 0.5$ \\
\hline G16 & $147.5+3.9$ & $118.3 \pm 6.3$ & $17.5 \pm 0.9$ & $16.2 \pm 1.6$ & $28.9 \pm 2.8$ & $79.4 \pm 1.7$ & $4.0 \pm 00$ & $18.9 \pm 0.9$ & $11.3 \pm 0.8$ & $20.6 \pm 2.5$ & $6.9 \pm 0.2$ \\
\hline G17 & $122.7+5.1$ & $101.2 \pm 7.4$ & $10.1 \pm 1.2$ & $11.4 \pm 1.2$ & $29.0 \pm 2.8$ & $84.4 \pm 1.7$ & $4.5 \pm 0.1$ & $17.7 \pm 0.6$ & $11.9 \pm 0.4$ & $20.4 \pm 1.1$ & $7.1 \pm 0.4$ \\
\hline G18 & $139.5+4.0$ & $117.8 \pm 4.0$ & $21.2 \pm 0.9$ & $20.4 \pm 2.0$ & $30.7 \pm 2.3$ & $66.1 \pm 0.1$ & $4.0 \pm 00$ & $19.4 \pm 1.8$ & $11.0 \pm 0.6$ & $21.0 \pm 1.2$ & $7.9 \pm 0.5$ \\
\hline G19 & $143.8+1.9$ & $146.2 \pm 6.7$ & $16.7 \pm 0.6$ & $23.4 \pm 3.5$ & $30.2 \pm 3.2$ & $66.7 \pm 1.6$ & $4.0 \pm 00$ & $21.5 \pm 1.2$ & $11.0 \pm 0.6$ & $18.7 \pm 1.5$ & $7.3 \pm 0.0$ \\
\hline G20 & $115.7+4.8$ & $121.9 \pm 3.9$ & $14.1 \pm 1.0$ & $21.9 \pm 2.8$ & $28.7 \pm 3.3$ & $62.8 \pm 1.7$ & $4.0 \pm 00$ & $20.2 \pm 1.2$ & $12.4 \pm 1.4$ & $22.2 \pm 2.3$ & $8.0 \pm 0.3$ \\
\hline G21 & $167.8+3.6$ & $125.9 \pm 6.8$ & $14.5 \pm 1.2$ & $28.1 \pm 3.1$ & $32.7 \pm 3.4$ & $80.0 \pm 1.1$ & $1.0 \pm 00$ & $47.2 \pm 2.0$ & $10.4 \pm 0.6$ & $32.4 \pm 1.5$ & $10.7 \pm 0.2$ \\
\hline $\mathrm{G} 22$ & $200.4+3.5$ & $143.9 \pm 3.0$ & $20.4 \pm 1.5$ & $34.4 \pm 3.5$ & $33.0 \pm 3.3$ & $86.1 \pm 1.4$ & $1.0 \pm 00$ & $23.2 \pm 1.3$ & $10.6 \pm 0.7$ & $29.2 \pm 1.8$ & $9.8 \pm 0.4$ \\
\hline G23 & $186.2+4.8$ & $129.7 \pm 5.4$ & $22.0 \pm 0.8$ & $40.7 \pm 4.7$ & $36.3 \pm 2.5$ & $82.2 \pm 1.8$ & $1.0 \pm 00$ & $24.0 \pm 1.8$ & $9.6 \pm 0.7$ & $35.9 \pm 2.8$ & $11.6 \pm 0.4$ \\
\hline G24 & $192.4+8.3$ & $136.1 \pm 8.3$ & $24.1 \pm 1.5$ & $28.5 \pm 2.9$ & $35.0 \pm 0.3$ & $80.5 \pm 2.4$ & $4.0 \pm 00$ & $20.5 \pm 1.3$ & $11.3 \pm 1.2$ & $26.5 \pm 1.3$ & $7.5 \pm 0.2$ \\
\hline G25 & $154.1+3.4$ & $134.5 \pm 5.4$ & $13.0 \pm 0.5$ & $13.7 \pm 2.7$ & $35.5 \pm 3.8$ & $83.3 \pm 1.1$ & $1.0 \pm 00$ & $25.1 \pm 0.9$ & $17.5 \pm 1.7$ & $35.9 \pm 2.4$ & $8.6 \pm 0.6$ \\
\hline G26 & $158.1+.3 .4$ & $140.8 \pm 3.8$ & $21.1 \pm 1.7$ & $25.0 \pm 2.8$ & $27.2 \pm 2.5$ & $75.0 \pm 2.0$ & $5.0 \pm 00$ & $17.6 \pm 1.3$ & $7.8 \pm 0.6$ & $26.9 \pm 1.5$ & $8.2 \pm 0.3$ \\
\hline
\end{tabular}


Table 3

Correlation coefficients between the main morphological characters in R. damascena genotypes

\begin{tabular}{|c|c|c|c|c|c|c|c|c|c|c|c|c|c|}
\hline Parameters & $\begin{array}{l}\text { Plant } \\
\text { height }\end{array}$ & $\begin{array}{l}\text { Plant } \\
\text { crown } \\
\text { diameter }\end{array}$ & $\begin{array}{l}\text { No of } \\
\text { main } \\
\text { stems } \\
\text { per } \\
\text { plant }\end{array}$ & $\begin{array}{l}\text { No of } \\
\text { nodes } \\
\text { in the } \\
\text { branch }\end{array}$ & $\begin{array}{l}\text { Internode } \\
\text { length }\end{array}$ & $\begin{array}{l}\text { Branches } \\
\text { angle }\end{array}$ & $\begin{array}{l}\text { Thorns } \\
\text { density }\end{array}$ & $\begin{array}{l}\text { Leaf } \\
\text { stipule } \\
\text { length }\end{array}$ & $\begin{array}{l}\text { Leaf } \\
\text { stipule } \\
\text { Width }\end{array}$ & $\begin{array}{l}\text { Peduncle } \\
\text { length }\end{array}$ & $\begin{array}{l}\text { Receptacle } \\
\text { length }\end{array}$ & $\begin{array}{l}\text { Flower } \\
\text { bud } \\
\text { length }\end{array}$ & $\begin{array}{l}\text { Flow } \\
\text { bud } \\
\text { diam }\end{array}$ \\
\hline $\begin{array}{l}\text { Plant } \\
\text { height }\end{array}$ & 1 & & & & & & & & & & & & \\
\hline $\begin{array}{l}\text { Plant } \\
\text { crown } \\
\text { diameter }\end{array}$ & $0.55^{\star \star}$ & 1 & & & & & & & & & & & \\
\hline $\begin{array}{l}\text { No of main } \\
\text { stems per } \\
\text { plant }\end{array}$ & $0.57^{\star \star}$ & $0.33^{* *}$ & 1 & & & & & & & & & & \\
\hline $\begin{array}{l}\text { No of } \\
\text { nodes in } \\
\text { the branch }\end{array}$ & $0.51^{\star \star}$ & $0.36^{\star \star}$ & $0.23^{\star \star}$ & 1 & & & & & & & & & \\
\hline $\begin{array}{l}\text { Internode } \\
\text { length }\end{array}$ & 0.17 ** & 0.12 & 0.05 & $0.16^{* *}$ & 1 & & & & & & & & \\
\hline $\begin{array}{l}\text { Branches } \\
\text { angle }\end{array}$ & 0.21 ** & 0.09 & -0.08 & 0.015 & 0.17 ** & 1 & & & & & & & \\
\hline $\begin{array}{l}\text { Thorns } \\
\text { density }\end{array}$ & $-0.47 \star \star$ & $-0.23^{\star \star}$ & -0.09 & -0.29 ** & $-0.19 * *$ & $-0.39 \star *$ & 1 & & & & & & \\
\hline $\begin{array}{l}\text { Leaf } \\
\text { stipule } \\
\text { length }\end{array}$ & 0.07 & 0.05 & -0.08 & 0.07 & 0.12 & 0.07 & -0.01 & 1 & & & & & \\
\hline $\begin{array}{l}\text { Leaf } \\
\text { stipule } \\
\text { Width }\end{array}$ & -0.05 & 0.04 & -0.04 & -0.07 & 0.04 & -0.04 & 0.07 & -0.05 & 1 & & & & \\
\hline $\begin{array}{l}\text { Peduncle } \\
\text { length }\end{array}$ & $0.45^{\star \star}$ & $0.36^{* \star}$ & 0.18 ** & 0.28 ** & 0.07 & 0.24 ** & $-0.56^{\star \star}$ & 0.08 & 0.05 & 1 & & & \\
\hline $\begin{array}{l}\text { Receptacle } \\
\text { length }\end{array}$ & 0.07 & -0.01 & -0.04 & 0.17 & 0.06 & $0.23^{\star \star}$ & $-0.36^{\star \star}$ & 0.06 & -0.16 & 0.18 ** & 1 & & \\
\hline $\begin{array}{l}\text { Flower bud } \\
\text { length }\end{array}$ & $0.46^{\star \star}$ & $0.27^{\star \star}$ & 0.11 & $0.21^{\star *}$ & 0.21 ** & 0.27 ** & $-0.56^{\star \star}$ & 0.06 & -0.04 & 0.36 ** & $0.27 \star \star$ & 1 & \\
\hline $\begin{array}{l}\text { Flower bud } \\
\text { diameter }\end{array}$ & -0.09 & -0.01 & -0.01 & 0.00 & -0.10 & -0.02 & $0.27 \star \star$ & -0.09 & 0.06 & -0.11 & 0.05 & -0.04 & 1 \\
\hline $\begin{array}{l}\text { No of } \\
\text { petals }\end{array}$ & $-0.27 \star \star$ & -0.16 & -0.10 & $-0.20 * \star$ & -0.03 & -0.11 & $0.39 * *$ & -0.10 & $0.17^{\star \star}$ & $-0.31^{\star *}$ & 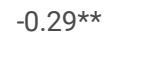 & $-0.35^{\star \star}$ & 0.07 \\
\hline
\end{tabular}


Table 4

Color indices of petals of 26 Damask rose landraces (mean \pm SE)

\begin{tabular}{|lllll|}
\hline Genotype & $\mathrm{L}^{*}$ & $\mathrm{a} *$ & $\mathrm{~b} *$ & Chroma \\
\hline G1 & $61.4 \pm 4.4$ & $31.59 \pm 3.6$ & $-0.4 \pm 0.4$ & $38.6 \pm 1.0$ \\
\hline G2 & $37.3 \pm 0.7$ & $52.41 \pm 2.1$ & $8.8 \pm 2.4$ & $41.9 \pm 4.1$ \\
\hline G4 & $65.0 \pm 3.2$ & $32.5 \pm 5.6$ & $-5.0 \pm 2.1$ & $39.9 \pm 0.7$ \\
\hline G5 & $49.0 \pm 1.7$ & $47.8 \pm 5.0$ & $-1.8 \pm 0.7$ & $53.9 \pm 2.1$ \\
\hline G6 & $67.2 \pm 1.2$ & $23.4 \pm 6.5$ & $24.7 \pm 3.8$ & $41.3 \pm 3.0$ \\
\hline G7 & $70.8 \pm 1.4$ & $26.5 \pm 1.9$ & $-0.9 \pm 0.5$ & $32.5 \pm 3.1$ \\
\hline G8 & $71.6 \pm 1.4$ & $28.1 \pm 2.4$ & $0.0 \pm 0.6$ & $34.1 \pm 5.1$ \\
\hline G9 & $74.8 \pm 1.1$ & $22.0 \pm 3.4$ & $22.3 \pm 1.5$ & $38.2 \pm 2.9$ \\
\hline G10 & $76.4 \pm 3.3$ & $27.5 \pm 5.3$ & $-3.1 \pm 0.9$ & $43.1 \pm 2.5$ \\
\hline G11 & $64.3 \pm 1.0$ & $36.3 \pm 0.6$ & $-3.6 \pm 0.3$ & $42.5 \pm 3.0$ \\
\hline G12 & $67.0 \pm 1.9$ & $25.9 \pm 2.2$ & $4.5 \pm 1.6$ & $31.3 \pm 0.6$ \\
\hline G13 & $59.5 \pm 3.1$ & $29.4 \pm 2.8$ & $-2.5 \pm 0.5$ & $36.5 \pm 1.0$ \\
\hline G14 & $55.9 \pm 5.1$ & $42.1 \pm 2.3$ & $-1.6 \pm 1.7$ & $49.1 \pm 2.0$ \\
\hline G15 & $60.6 \pm 3.3$ & $37.3 \pm 4.5$ & $-0.9 \pm 3.0$ & $44.3 \pm 3.2$ \\
\hline G16 & $41.7 \pm 1.7$ & $53.0 \pm 1.8$ & $-8.8 \pm 0.8$ & $60.7 \pm 4.0$ \\
\hline G17 & $63.2 \pm 2.9$ & $34.4 \pm 4.0$ & $-2.5 \pm 0.5$ & $41.5 \pm 0.4$ \\
\hline G18 & $43.6 \pm 5.1$ & $37.0 \pm 2.9$ & $1.6 \pm 0.6$ & $43.0 \pm 7.9$ \\
\hline G19 & $39.0 \pm 2.4$ & $35.0 \pm 2.7$ & $3.3 \pm 1.8$ & $42.2 \pm 6.3$ \\
\hline G20 & $55.8 \pm 4.9$ & $35.7 \pm 7.6$ & $3.7 \pm 3.4$ & $41.9 \pm 4.5$ \\
\hline G21 & $60.2 \pm 0.2$ & $25.7 \pm 5.0$ & $2.0 \pm 0.4$ & $32.8 \pm 1.9$ \\
\hline G22 & $66.2 \pm 3.9$ & $29.9 \pm 6.5$ & $-0.6 \pm 1.6$ & $36.9 \pm 0.2$ \\
\hline G23 & $65.7 \pm 1.5$ & $24.3 \pm 3.0$ & $-2.2 \pm 0.8$ & $41.3 \pm 3.7$ \\
\hline G24 & $66.3 \pm 2.9$ & $32.1 \pm 3.4$ & $0.3 \pm 2.7$ & $39.1 \pm 1.4$ \\
\hline G26 & $65.4 \pm 3.1$ & $33.1 \pm 3.1$ & $1.4 \pm 1.4$ & $40.1 \pm 2.1$ \\
\hline
\end{tabular}

\section{Essential oil components}

Significant differences were found between chromatographic characteristics of the genotypes, indicating differences in their chemical compositions. In total, twenty-five compounds were identified in extracted oils of fresh petals of selected 26 Iranian genotypes (Table 5). The principal components of the essential oils were n-heneicosane, citronellol, and nonadecane in all genotypes. Results show that geraniol is the highest component in Damask rose oil, except in G14 and G18. The highest concentration of geraniol was found in G5 (27.76\%), G9 (27.33\%), and G2 (27.27\%) respectably. Geraniol has been reported to be one of the main essential oil components in Damask rose [5]. According to GC-MS results, the highest nonadecane contents $(42.51 \%, 35.06 \%, 30.91 \%$, and $30.26 \%)$ were found in essential oils of G12, G14, G11, and G19, respectively. Several studies indicated that heneicosane, heptadecane, nonadecane, and eicosane were abundant hydrocarbons in rose oil [32]. Based on the results, G14 (34.69\%), G23 (30.92\%), and G21 (30.82\%) had the highest content of heneicosane. In the current study, damascone ( $\mathrm{Z}$ )-a and $\beta$-damascenone had been determined in most of the genotypes, but $\beta$-damascone was less abundant. The highest concentrations of damascone (Z)- $\alpha(2.88 \%), \beta$-damascone (0.96\%), and $\beta$-damascenone (1.76\%) were found in G20, G3, and G3, respectively (Table 5). Damascone (Z)-a, $\beta$-damascone, and $\beta$-damascenone are the trace components and quality markers for Damask rose oil, playing an important organoleptic role in rose oil [40]. The highest quantity of $\beta$-citronellol (40\%) was recorded in $\mathrm{G} 26$ which is one of the most abundant acyclic terpenes in rose oil. 
Table 5

Quantitative data for the twenty-five components of the essential oil content (\%) from 26 Damask roses genotypes, determined by GC- $\mathrm{A}$

\begin{tabular}{|c|c|c|c|c|c|c|c|c|c|c|c|c|c|c|c|}
\hline No & Compound & $\mathrm{Rt}^{\mathrm{a}}$ & $\mathrm{LRI}^{\mathrm{b}}$ & $\mathrm{RI}^{\mathrm{c}}$ & G1 & G2 & G3 & G4 & G5 & G6 & G7 & G8 & G9 & G10 & $\overline{\mathbf{G 1}}$ \\
\hline 1 & $\begin{array}{l}\text { Phenyl ethyl } \\
\text { alcohol }\end{array}$ & 11.75 & 1106 & 1111 & - & 0.334 & - & - & 0.309 & 1.541 & - & 0.302 & 0.400 & 0.240 & - \\
\hline 2 & $\begin{array}{l}\text { Dihydro } \\
\text { linalool }\end{array}$ & 12.82 & 1131 & 1145 & 0.556 & 1.451 & - & 1.057 & 0.826 & 41.189 & 0.388 & 0.831 & 1.271 & 0.716 & - \\
\hline 3 & $\beta$-Citronellol & 16.80 & 1223 & 1225 & 34.660 & 29.268 & 14.911 & 37.574 & 21.812 & 15.608 & 27.728 & 31.830 & 30.389 & 30.77 & 10 \\
\hline 4 & Neral & 17.32 & 1235 & 1237 & 2.281 & 10.258 & 0 & 3.893 & 7.347 & 6.221 & 2.239 & 3.791 & 10.832 & 3.914 & 0 \\
\hline 5 & Geraniol & 17.95 & 1249 & 1250 & 6.182 & 27.270 & 2.931 & 12.182 & 27.768 & 7.986 & 6.935 & 10.863 & 27.335 & 10.364 & $2 .:$ \\
\hline 6 & $\begin{array}{l}\text { Linalyl } \\
\text { acetate }\end{array}$ & 18.16 & 1254 & 1257 & 0.220 & - & - & - & 0.470 & - & - & - & 0.291 & 0.277 & - \\
\hline 7 & Geranial & 18.62 & 1264 & 1267 & 0.202 & 0.877 & - & 0.332 & 0.363 & - & - & - & 0.728 & 0.268 & - \\
\hline 8 & $\begin{array}{l}\text { Dihydro } \\
\text { citronellol } \\
\text { acetate }\end{array}$ & 21.04 & 1319 & 1320 & 0.232 & 0.454 & - & 0.278 & 0.408 & - & - & - & 0.360 & 0.242 & - \\
\hline 9 & $\begin{array}{l}\text { Damascone } \\
\text { (Z)-a }\end{array}$ & 22.64 & 1355 & 1358 & 0.368 & 1.847 & - & 0.538 & 2.226 & 1.666 & 0.487 & 0.394 & 1.281 & 0.466 & - \\
\hline 10 & $\begin{array}{l}\text { Damascenone } \\
\text { (E) }-\beta\end{array}$ & 23.87 & 1383 & 1384 & 0.762 & - & 1.766 & 0.526 & 0.308 & 1.761 & 0.704 & 0.812 & 0.274 & 0.768 & 1.: \\
\hline 11 & $\begin{array}{l}\text { Damascone } \\
\text { (E)- } \beta\end{array}$ & 25.17 & 1413 & 1414 & - & 0.528 & 0.967 & - & - & - & - & - & 0.253 & 0.299 & $0 . !$ \\
\hline 12 & $\begin{array}{l}\text { Dodecen-1-ol } \\
\text { (2E) }\end{array}$ & 27.55 & 1469 & 1471 & - & 0.717 & - & - & - & - & - & 0.736 & 2.099 & 0.199 & $0 . \therefore$ \\
\hline 13 & n Heptadecane & 36.74 & 1700 & 1700 & 1.979 & 0.435 & 1.315 & 2.311 & 1.609 & - & 1.271 & 0.583 & 0.529 & 1.923 & 1.1 \\
\hline 14 & z z-Farnesol & 36.68 & 1698 & 1698 & 0.638 & 1.026 & 0.503 & - & - & - & - & 0.584 & 1.367 & 0.432 & - \\
\hline 15 & $\begin{array}{l}\text { (e e)-Farnesyl } \\
\text { acetate }\end{array}$ & 42.01 & 1845 & 1846 & 0.845 & 4.002 & - & 1.138 & 2.249 & - & 1.173 & 1.399 & 3.756 & 1.295 & $0 .:$ \\
\hline 16 & n-Octadecane & 40.40 & 1800 & 1800 & - & 0.360 & 0.567 & 0.262 & - & - & - & - & 0.429 & - & 0.4 \\
\hline 17 & $\begin{array}{l}\mathrm{n}- \\
\text { Hexadecanol }\end{array}$ & 43.03 & 1874 & 1875 & 2.918 & 0.317 & 4.184 & 5.202 & 3.815 & 1.174 & 2.634 & 0.605 & 0.343 & 3.308 & 3.4 \\
\hline 18 & Nonadecane & 43.92 & 1900 & 1903 & 27.229 & 5.580 & 28.927 & 19.81 & 14.812 & 5.455 & 23.574 & 10.710 & 4.347 & 23.396 & 30 \\
\hline 19 & n-Eicosane & 47.33 & 2000 & 2004 & 2.587 & 1.362 & 4.330 & 1.850 & 1.542 & - & 3.260 & 2.646 & 1.242 & 2.488 & 4.8 \\
\hline 20 & $\begin{array}{l}\mathrm{n}- \\
\text { Heneicosane }\end{array}$ & 50.47 & 2100 & 2100 & 13.860 & 7.729 & 21.574 & 9.429 & 9.522 & 4.784 & 21.243 & 19.543 & 6.749 & 13.208 & 27 \\
\hline 21 & n-Docosane & 53.54 & 2200 & 2195 & 0.286 & 0.405 & 0.724 & - & 0.224 & 9.335 & 0.578 & 0.749 & 0.342 & 0.310 & 0.8 \\
\hline 22 & n-Tricosane & 56.48 & 2300 & 2303 & 3.236 & 4.874 & 6.506 & 2.480 & 3.295 & 2.635 & 6.039 & 11.177 & 3.905 & 3.908 & 7.1 \\
\hline 23 & e-e Farnesol & 38.30 & 1742 & 1743 & 0.363 & 0.522 & 0.439 & - & - & - & 0.359 & 0.524 & 0.861 & - & $0 . \therefore$ \\
\hline 24 & Phytol & 45.37 & 1942 & 1943 & - & - & - & - & - & - & - & 0.311 & - & - & 3.: \\
\hline \multirow[t]{2}{*}{25} & $\begin{array}{l}\text { Trans rose } \\
\text { oxide }\end{array}$ & 12.45 & 1122 & 1125 & - & - & 0.605 & - & - & - & - & - & - & - & $0 . !$ \\
\hline & & Total & & & 99.413 & 99.625 & 90.256 & 98.873 & 98.912 & 99.360 & 98.615 & 98.400 & 99.393 & 98.801 & 97 \\
\hline
\end{tabular}

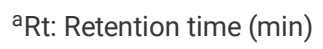

bLRI: RI from literature (Adams, 2017).

${ }^{\mathrm{C}} \mathrm{RI}$ : Experimentally determined retention index (RI) 
Table 5

(continue). Quantitative data for the twenty-five components of the essential oil content (\%) from 26 Damask rose genotypes, determin

\begin{tabular}{|c|c|c|c|c|c|c|c|c|c|c|c|c|c|c|c|}
\hline No & Compound & $\mathbf{R t}^{\mathbf{a}}$ & LRI $^{b}$ & $\mathrm{R}^{\mathbf{c}}$ & G14 & G15 & G16 & G17 & G18 & G19 & G20 & G21 & G22 & G23 & $\bar{G}$ \\
\hline 1 & $\begin{array}{l}\text { Phenyl ethyl } \\
\text { alcohol }\end{array}$ & 11.75 & 1106 & 1111 & 0 & 0.206 & 0.224 & - & - & 0.103 & 0.187 & - & 0.165 & 0.409 & 0. \\
\hline 2 & $\begin{array}{l}\text { Dihydro } \\
\text { linalool }\end{array}$ & 12.82 & 1131 & 1145 & 2.559 & 10.647 & 3.064 & 4.434 & 23.869 & 0.553 & 0.989 & - & 0.723 & 0.484 & $0 . ؟$ \\
\hline 3 & $\beta$-Citronellol & 16.80 & 1223 & 1225 & 6.155 & 29.638 & 15.961 & 18.993 & 14.855 & 33.163 & 19.397 & 11.808 & 29.668 & 14.940 & 12 \\
\hline 4 & Neral & 17.32 & 1235 & 1237 & - & 3.675 & 5.110 & 0.734 & - & 0.414 & 3.549 & 2.867 & 3.018 & 0.308 & $1 .^{\circ}$ \\
\hline 5 & Geraniol & 17.95 & 1249 & 1250 & - & 9.394 & 12.120 & 5.707 & - & 2.211 & 13.797 & 6.415 & 19.244 & 4.208 & 10 \\
\hline 6 & $\begin{array}{l}\text { Linalyl } \\
\text { acetate }\end{array}$ & 18.16 & 1254 & 1257 & - & 0.692 & 2.762 & - & - & - & 2.377 & - & 0.152 & - & 0. \\
\hline 7 & geranial & 18.62 & 1264 & 1267 & - & 0.219 & 0.285 & - & - & - & - & - & - & - & - \\
\hline 8 & $\begin{array}{l}\text { Dihydro } \\
\text { citronellol } \\
\text { acetate }\end{array}$ & 21.04 & 1319 & 1320 & - & - & 0.320 & - & - & 0.221 & 0.471 & - & 0.180 & - & 0. \\
\hline 9 & $\begin{array}{l}\text { Damascone } \\
\text { (Z)-a }\end{array}$ & 22.64 & 1355 & 1358 & - & 0.314 & 2.081 & - & 1.768 & 0.233 & 2.880 & - & 0.571 & - & - \\
\hline 10 & $\begin{array}{l}\text { Damascenone } \\
(E)-\beta\end{array}$ & 23.87 & 1383 & 1384 & - & 0.594 & 0.247 & 0.885 & - & 0.785 & 0.199 & 0.415 & 0.775 & 0.979 & $0 . a^{\prime}$ \\
\hline 11 & $\begin{array}{l}\text { Damascone } \\
\text { (E)- } \beta\end{array}$ & 25.17 & 1413 & 1414 & - & 0.592 & 1.270 & 0.418 & - & 0.170 & - & - & 0.166 & 0.519 & - \\
\hline 12 & $\begin{array}{l}\text { Dodecen-1-ol } \\
\text { (2E) }\end{array}$ & 27.55 & 1469 & 1471 & - & - & - & 0.423 & 1.396 & 0.195 & - & - & 0.144 & - & 0. \\
\hline 13 & Heptadecane & 36.74 & 1700 & 1700 & 0.854 & 0.569 & 1.459 & 0.76 & 1.300 & 2.532 & 0.644 & 1.174 & 1.296 & 0.545 & $2 .^{\circ}$ \\
\hline 14 & z z-Farnesol & 36.68 & 1698 & 1698 & - & - & 1.749 & 3.017 & - & - & 0.364 & - & - & 0.707 & 0. \\
\hline 15 & $\begin{array}{l}\text { (e e)-Farnesyl } \\
\text { acetate }\end{array}$ & 42.01 & 1845 & 1846 & - & - & 2.834 & 0.870 & - & 0.224 & 2.279 & 0.603 & 1.534 & 0.336 & $1 . \therefore$ \\
\hline 16 & n-Octadecane & 40.40 & 1800 & 1800 & - & - & - & - & 2.214 & - & - & - & - & - & - \\
\hline 17 & $\begin{array}{l}\mathrm{n}- \\
\text { Hexadecanol }\end{array}$ & 43.03 & 1874 & 1875 & 2.386 & 0.497 & 1.386 & 1.119 & 9.166 & 3.829 & 1.270 & 2.033 & 2.302 & 1.979 & 1.4 \\
\hline 18 & Nonadecane & 43.92 & 1900 & 1903 & 35.061 & 9.54 & 19.044 & 25.131 & 12.882 & 30.264 & 14.43 & 29.524 & 18.540 & 22.789 & 20 \\
\hline 19 & n-Eicosane & 47.33 & 2000 & 2004 & 5.811 & 1.797 & 1.9154 & 3.975 & 1.821 & 2.919 & 2.323 & 4.193 & 2.212 & 4.469 & 3.6 \\
\hline 20 & $\begin{array}{l}\text { n- } \\
\text { Heneicosane }\end{array}$ & 50.47 & 2100 & 2100 & 34.695 & 13.389 & 16.763 & 24.094 & 11.685 & 15.751 & 18.080 & 30.824 & 13.986 & 30.925 & 20 \\
\hline 21 & n-Docosane & 53.54 & 2200 & 2195 & 1.065 & 0.607 & 0.590 & 0.704 & 0 & 0.346 & 0.824 & 0.718 & 0.330 & 1.056 & 0.4 \\
\hline 22 & n-Tricosane & 56.48 & 2300 & 2303 & 10.017 & 8.290 & 10.170 & 6.019 & 5.288 & 4.215 & 14.205 & 8.920 & 3.575 & 11.113 & $4 .^{\circ}$ \\
\hline 23 & e-e Farnesol & 38.30 & 1742 & 1743 & 0 & 8.282 & 0 & 0 & & 0 & 0 & 0 & 0 & 0 & $0 . \AA$ \\
\hline 24 & Phytol & 45.37 & 1942 & 1943 & & 0 & 0 & 1.059 & & 0 & 0 & 0 & 0 & 3.375 & 0 \\
\hline \multirow[t]{2}{*}{25} & $\begin{array}{l}\text { Trans rose } \\
\text { oxide }\end{array}$ & 12.45 & 1122 & 1125 & 0 & 0.208 & 0 & 0 & 0 & 0.187 & 0 & 0 & 0.100 & 0.439 & 0 \\
\hline & & Total & & & 98.605 & 98.660 & 99.362 & 98.348 & 86.255 & 98.322 & 98.281 & 99.498 & 98.690 & 99.584 & 79 \\
\hline
\end{tabular}

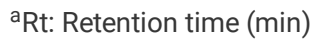

bLRI: RI from literature (Adams, 2017).

${ }^{\mathrm{C}} \mathrm{Rl}$ : Experimentally determined

In the current study, neral was present in all genotypes, except in G3, G11, G14, and G18. A major concentration of neral was in G9 (10.83\%) and G2 (10.25\%). Geranial was found in G1, G2, G4, G5, G9, G10, G15, and G16 genotypes at low levels (Table 5). Neral and geranial are citral isomers, which have been found in Damask rose essential oil ${ }^{[28]}$. Farnesol, natural sesquiterpene alcohol in essential oils, was found to have the potential for alleviating massive inflammation, oxidative stress, and lung injury [41,42]. Farnesol has been widely used in cosmetics, pharmaceuticals, industrial materials, and as a material for carotenoid 
and tocopherol [43]. Farnesol is a sesquiterpene trans and exists in some Damask genotypes, a higher amount of it was found in G17 (3.01\%) and the highest e-e Farnesol was observed in G15 (8.28\%).

Rose oxide is an insignificant component of rose oil [44]. In this study, the rose oxide has been found in low concentrations in G3, G11, G15, G19, G22, G23, and G26 (Table 5). Phenethyl alcohol is an enjoyable floral perfume belonging to aromatic alcohols, and one of the main components of rose hydrosols which mainly is used in perfumery [2]. However, this compound was detected at low levels only in some of the selected Damask rose genotypes including G6 (1.54\%), G23 (0.40\%), and G2 (0.33\%). Phytol is one of the major components of plant-derived essential oils which has been recognized for its wide range of pharmacological effects on the nervous system, including anxiolytic, antidepressant, and antimicrobial [45-47]. Several recent studies have suggested that some phytol-derivatives (phytanol, phytanyl amine, and phytanyl mannose) by induction of the expression of a range of chemokines and cytokines effects target tumor cells $[48,49]$. Other hydrocarbon-like ingredients, $n$-docosane and $n$-tricosane, were also identified in Damask roses essential oil. Quantities of $n$ tricosane were much more than that of n-docosane in all genotypes. In the present study, G6 (9.33\%) and G20 (14.20\%) genotypes showed the highest contents of n-tricosane and $n$-docosane, respectively.

\section{Discussion}

Several studies have been conducted to date on the genetic diversity of $R$. damascena in Iran, which has shown a high diversity and genetic variation of this species $[6,8,50]$. In this study, $R$. damascena genotypes showed a remarkable diversity in petal color from dark pink (G3/Tehran genotype) to pale pink (G9/Fars genotype) and white (G2/ Isfahan and G26/ East Azarbayjan genotypes). However, the majority of them were pink or pinkish (Fig. 3; Table 4). Some anthocyanins such as pelargonidin and cyanidin in the petal cells are responsible for the color of rose flowers [51]. Petals of industrial oil-bearing damask roses grown in the world are typically pink, while wild roses usually have pink or white flowers [52]. Karami et al [33] reported a positive relationship between essential oil content and anthocyanin concentration in Damask rose.

The number of petals is a very important indicator of the total essential oil. Significant negative correlations between thorn density and morphological characters, excluding bud diameter, were observed. Additionally, there was a significant positive correlation ( $0.39 * \star)$ between the number of petals and thorn density (Table 3). Therefore, it is possible to select genotypes with a higher flower weight and number of flowers in attempts to improve the flower yield and essential oil content [7, 32].

According to the results (Fig. 1, Fig. 2), harvesting time had a major effect on essential oil content, and the morning time harvested flowers had a higher essential oil content. Also, there is no positive relation between oil content and petal number. This is consistent with some reports that the oil content of the damask rose flowers depended on the time of harvesting, and the petals harvested in the morning time had a higher oil content [5,23]. Results of the current study also showed that the essential oil content was influenced by harvesting time in the majority of 26 genotypes of the Damask roses, confirming that morning time was the best time to harvest petals, which is similar to some other reports [53-56].

Large differences were observed between 26 selected Damask rose genotypes for the content of essential oils (Table 5) which was in agreement with the results of researches where reported high variations in the volatile compounds of Damask rose oil [11, 18, 32]. It has been reported that the quantity and composition of essential oil ingredients are significantly influenced by the genotype and agronomic conditions, as well as plant and flower developmental stage and harvesting time [57-59]. Overall, the content of monoterpenes (citronellol, nerol, and E-geraniol), sesquiterpenes, and aliphatic hydrocarbons was high (Table 5). Furthermore, e-geraniol, one of the major rose-oil components, was high in all 26 selected Damask rose genotypes. The percentage of four major hydrocarbons (heptadecane, nonadecane, eicosane, and heneicosane) were also high in the extracted essential oils (Table 5). Similar to other reports, this study revealed high variations between Rosa damascena genotypes regarding oil content and components, morphological diversity, and petal color [9, $60-63]$.

\section{Conclusions}

Results from this study revealed that Damask rose genotypes in Iran have significant diversity in morphological characters, oil content, and also composition. The harvesting time of Damask rose flowers significantly affected the essential oil yield, and for most genotypes harvesting recommended to be performed in the morning, but for higher oil content of G2 and G5 genotypes, evening harvesting time might be recommended. The varied deviations in petal colors, petal numbers, and essential oil content in genotypes were observed in this experiment. Thus, the existencof these characters and a good chemical variation shown in profiling reveals that the studied collection of Damask rose is a good source for the selection of the industrial oil-bearing damask rose cultivars and those that could be used as an ornamental plant in the landscape because of its unique scented flowers. Compared with the other genotypes, G5 and G21 had the highest essential oil content. 25 volatile compounds were identified in the essential oil of Damask rose genotypes. The highest concentration of geraniol, $\beta$ citronellol, nonadecane, and $\beta$-damascenone were found in G12 (42.51\%), G26 (40.82\%), G5 (27.76\%), and G3 (1.76\%) genotypes, respectively. It has been found that the most abundant compounds are of several main classes including alcohols (citronellol, geraniol, nerol) and hydrocarbons (heptadecane, nonadecane, eicosane, and heneicosane). As a final conclusion, results revealed that the morphological and biochemical diversity of Damask rose genotypes can be used effectively to characterize genetic diversity between different genotypes and to select special traits in the breeding program.

\section{Declarations}

\section{Acknowledgements}

Not applicable 
$\mathrm{MO}, \mathrm{AKH}$, and $\mathrm{MK}$ conceived and designed the study; $\mathrm{MO}$ and $\mathrm{ASH}$ contributed to literature research; $\mathrm{MO}$ performed the experiments and collected the results; $\mathrm{OR}$ and $\mathrm{MO}$ analyzed and interpreted the data; $\mathrm{MO}$ and $\mathrm{MK}$ were major contributors in writing the manuscript; $\mathrm{ZZ}, \mathrm{AKH}$ and $\mathrm{MK}$ guided all aspects of the research project and revised the manuscript; All authors read and approved the final manuscript.

\section{Funding}

This work was supported by Department of Horticulture Science, College of Agriculture and Natural Resources, University of Tehran, Karaj 31587, Iran

\section{Availability of data and materials}

All data generated or analysed during this study are included in this published article.

\section{Ethics approval and consent to participate}

Not applicable.

\section{Consent for publication}

Not applicable.

\section{Competing interests}

The authors declare that they have no competing interests.

\section{References}

1. Iwata H, Kato T, Ohno S. Triparental origin of Damask roses. Gene. 2000; 259(2): 53-59. https://doi.org/10.1016/S0378-1119(00)00487-X

2. Moein M, Zarshenas MM, Delnavaz S. Chemical composition analysis of rose water samples from Iran. Pharma Biol. 2014; 52(10): 1358-1361. https://doi.org/10.3109/13880209.2014.885062

3. Moein M, Etemadfard H, Zarshenas MM. Investigation of different Damask rose (Rosa damascena Mill.) oil samples from traditional markets in Fars (Iran); focusing on the extraction method. Trends in Pharma Sci. 2016; 2(1): 51-58.

4. Rusanov K, Kovacheva N, Vosman B, Zhang L, Rajapakse S, Atanassov A, Atanassov I. Microsatellite analysis of Rosa damascena Mill. genotypes reveal genetic similarity between genotypes used for rose oil production and old Damask rose varieties. Theo and Applied Genetics. 2005; 111(4): 804-809. https://doi.org/10.1007/s00122-005-2066-9

5. Baydar H, Baydar NG. The effects of harvest date, fermentation duration and Tween 20 treatment on essential oil content and composition of industrial oil rose (Rosa damascena Mill.). Ind.Crops Prod. 2005; 21(2): 251-255. https://doi.org/10.1016/S0926-6690(04)00056-1

6. Babaei A, Tabaei-Aghdaei SR, Khosh-Khui M, Omidbaigi R, Naghavi MR, Esselink GD, Smulders MJ. Microsatellite analysis of Damask rose (Rosa damascena Mill.) genotypes from various regions in Iran reveals multiple genotypes. BMC Plant Biol. 2007; 7(1): 1-6. https://doi.org/10.1186/1471-22297-12

7. Tabaei-Aghdaei SR, Babaei A, Khosh-Khui M, Jaimand K, Rezaee MB, Assareh MH, Naghavi MR. Morphological and oil content variations amongst Damask rose (Rosa damascena Mill.) landraces from different regions of Iran. Sci Horti. 2007; 113(1): 44-48. https://doi.org/10.1016/j.scienta.2007.01.010

8. Kiani M, Zamani Z, Khalighi A, Fatahi R, Byrne DH. Wide genetic diversity of Rosa damascena Mill. germplasm in Iran as revealed by RAPD analysis. Sci Horti. 2008; 115(4): 386-392. https://doi.org/10.1016/j.scienta.2007.10.013

9. Kazaz S, Erbas S, Baydar H, Dilmacunal T, Koyuncu MA. Cold storage of oil rose (Rosa damascena Mill.) flowers. Sci Horti. 2010; 126(2): 284-290. https://doi.org/10.1016/j.scienta.2010.06.018

10. Nasri F, Fadakar A, Yousefi B, Zahedi B. Evaluation of genetic diversity of some Damask rose (Rosa damascena Mill.) genotypes of Kurdistan province using morphological traits. J of Ornamental Plants. 2016; 6(4): 237-243.

11. Sharma S, Kumar, R. Effect of temperature and storage duration of flowers on essential oil content and composition of Damask rose (Rosax damascena Mill.) under western Himalayas. J. of Applied Res on Med and Aroma Plants. 2016; 3(1): 10-17. https://doi.org/10.1016/j.jarmap.2015.10.001

12. Kovacheva N, Rusanov K, Atanassov I. Industrial cultivation of oil bearing rose and rose oil production in Bulgaria during 21 st century, directions and challenges. Biotech \& Biotech Equip. 2010;24(2): 1793-1798. https://doi.org/10.2478/V10133-010-0032-4

13. Global"Rose Essential Oil Market"2019 Industry Research Report. Market research report. 2019; p.80.

14. Kaul K, Karthigeyan S, Dhyani D, Kaur N, Sharma RK, Ahuja PS. Morphological and molecular analyses of Rosa damascenax R. bourboniana interspecific hybrids. Sci Horti. 2009; 122(2): 258-263. https://doi.org/10.1016/j.scienta.2009.05.027

15. Boskabady MH, Shafei MN, Saberi Z, Amini S. Pharmacological effects of Rosa damascena. I. J. B.M. Sci. 2011; 14(4): $295-307$.

16. Gorji-Chakespari A, Nikbakht AM, Sefidkon F, Ghasemi-Varnamkhasti M, Valero EL. Classification of essential oil composition in Damask Rose Mill. genotypes using an electronic nose. J. of Applied Res on Medi and Aroma Plants. 2017; 4: 27-34. https://doi.org/10.1016/j.jarmap.2016.07.004

17. Dobreva A, Kovacheva N. Daily dynamics of the essential oils of Rosa damascena Mill. and Rosa alba L. Agri Sci and Tech. 2010; 2(2): 71-74.

18. Rusanov K, Kovacheva N, Rusanova M, Atanassov I. Traditional Rosa damascena flower harvesting practices evaluated through GC/MS metabolite profiling of flower volatiles. Food Chem. 2011; 129(4): 1851-1859. https://doi.org/10.1016/j.foodchem.2011.05.132 
19. Pal PK, Singh RD. Understanding crop-ecology and agronomy of Rosa damascena Mill. for higher productivity. Aus J of Crop Sci. 2013;7(2): 196-205.

20. Koksal N, Aslancan H, Sadighazadi S, Kafkas E. Chemical investigation on Rosa damascena Mill. volatiles; Effects of storage and drying conditions. Acta Scie Polonorum: Hortorum Cultus. 2015; 14(1); 105-114.

21. Krupčík J, Gorovenko R, Špánik I, Sandra P, Armstrong DW. Enantioselective comprehensive two-dimensional gas chromatography. A route to elucidate the authenticity and origin of Rosa damascena Miller essential oils. Journal of Separation Scie. 2015; 38(19): 3397-3403.

https://doi.org/10.1002/jssc.201500744

22. Widrlechner MP. History and utilization of Rosa damascena. Economic Botany. 1981;35(1): 42-58. https://doi.org/10.1007/BF02859214

23. Rusanov K, Kovacheva N, Stefanova K, Atanassov A, Atanassov I. Rosa damascena-genetic resources and capacity building for molecular breeding. Biotech \& Biotech Equipt. 2009; 23(4): 1436-1439. https://doi.org/10.2478/V10133-009-0009-3

24. Erbas S, Baydar H. Variation in scent compounds of oil-bearing rose (Rosa damascena Mill.) produced by headspace solid phase microextraction, hydrodistillation and solvent extraction. Rec of Natural Prod. 2016; 10(5): 555-565.

25. Saint-Lary L, Roy C, Paris JP, Martin JF, Thomas OP, Fernandez X. Metabolomics as a tool for the authentication of rose extracts used in flavor and fragrance area. Metabolomics. 2016; 12(3): 49, 1-11. https://doi.org/10.1007/s11306-016-0963-3

26. Aydinli M, Tutaş M. Production of rose absolute from rose concrete. Flavor and Fragr. 2003; 18(1): 26-31. https://doi.org/10.1002/ffj.1138

27. Abdel-Hameed ESS, Bazaid SA, Hagag HA. Chemical characterization of Rosa damascena Miller var. Trigintipetala Dieck essential oil and it's in vitro genotoxic and cytotoxic properties. J.of Esse Oil Res. 2016; 28(2); 121-129. https://doi.org/10.1080/10412905.2015.1099120

28. Nedeltcheva-Antonova D, Stoicheva P, Antonov L. Chemical profiling of Bulgarian rose absolute (Rosa damascena Mill.) using gas chromatographymass spectrometry and trimethylsilyl derivatives. Ind. Crops Prod. 2017; 108: 36-43. https://doi.org/10.1016/j.indcrop.2017.06.007

29. Kiani M, Zamani Z, Khalighi A, Fatahi R, Kiani M. Collection and evaluation of morphological diversity of Damask rose genotypes of Iran. Iranian J of Horti Sci. 2011; 41: 223-233.

30. Yousefi B. Screening of Rosa damascena Mill. landraces for flower yield and essential oil content in cold climates. Folia Horticulturae. 2016; 28(1): 31-40. https://doi.org/10.1515/fhort-2016-0005

31. Mitrofanova I, Grebennikova O, Brailko V, Paliy A, Marko N, Lesnikova-Sedoshenko N, Mitrofanova O. Physiological and biochemical features of some cultivars in essential oil rose (Rosax damascena Mill.) growing in situ and in vitro. Int J of PharmTech Res. 2016; 9(7): $226-232$.

32. Baydar H, Erbas S, Kaza, S. Variations in floral characteristics and scent composition and the breeding potential in seed-derived oil-bearing roses (Rosa damascena Mill.). Turkish J. of Agri and Forestry. 2016; 40(4); 560-569. https://doi.org/10.3906/tar-1512-57

33. Karami A, Khosh-Khui M, Salehi H, Saharkhiz MJ, Rowshan V. Headspace analysis of floral scent from two distinct genotypes of Iranian Damask rose (Rosa damascena Mill.). J. of Esse Oil Bear Plant. 2013; 16(4): 489-498. https://doi.org/10.1080/0972060X.2013.813266

34. McGuire RG. Reporting of objective color measurements. Hort Sci. 1992; 27(12): 1254-1255. https://doi.org/10.21273/HORTSCI.27.12.1254

35. Khan MA, Rehman SU. Extraction and analysis of essential oil of Rosa species. Int. J. Agric. Biol. 2005; 7:973-974.

36. Shibamoto T. Retention Indices in Essential Oil Analysis (Vol. 259). Huethig Verlag, New York. 1987; p. $259-274$.

37. McLafferty FW, Stauffer DB. The Wiley NBS Registry of Mass Spectral Data; Wiley. New York. 1989; p. 518.

38. Adams RP. Identification of Essential Oils by Ion Trap Mass Spectroscopy. Academic press. San Diego, California. 2012 ; p. 302.

39. Meyer, R., Krueger, D. (2001). Minitab guide to statistics. Prentice Hall PTR.80 p

40. Ohloff G, Demole E. Importance of the odoriferous principle of Bulgarian rose oil in flavour and fragrance chemistry. Journal of Chroma. 1987; 406: 181183. https://doi.org/10.1016/S0021-9673(00)94029-9

41. Derengowski LS, De-Souza-Silva C, Braz SV, Mello-De-Sousa TM, Báo SN, Kyaw CM, Silva-Pereira I. Antimicrobial effect of farnesol, a Candida albicans quorum sensing molecule, on Paracoccidioides brasiliensis growth and morphogenesis. Annals of Clinical Microbiology and Antimicrobials. 2009; 8(1): 13. https://doi.org/10.1186/1476-0711-8-13

42. Hammer KA, Carson CF. Antibacterial and antifungal activities of essential oils. Lipids and essential oils as antimicrobial agents. 2011; 255-306.

43. Clarke S. Chapter 3-Families of compounds that occur in essential oils. Essential Chemistry for Aromatherapy. 2008; 41-77.

44. Leffingwell JC, Leffingwell D. GRAS flavor chemicals-detection thresholds. Perfumer \& Flavorist. 1991;16(1): 1-19.

45. Murbach Teles Andrade BF, Nunes Barbosa L, da Silva Probst I, Fernandes Júnior A. Antimicrobial activity of essential oils. Journal of Essential Oil Research. 2014; 26(1): 34-40. https://doi.org/10.1080/10412905.2013.860409

46. Pereira JC, Mario LRJ. Phytol a Natural Diterpenoid with Pharmacological Applications on Central Nervous System: A Review. Recent patents on biotechnology. 2014; 8(3): 194-205.

47. Gutbrod K, Romer J, Dörmann P. Phytol metabolism in plants. Progress in lipid research. 2019; 74: 1-17. https://doi.org/10.1016/j.plipres.2019.01.002

48. Aachoui Y, Chowdhury RR, Fitch RW, Ghosh SK. Molecular signatures of phytol-derived immunostimulants in the context of chemokine-cytokine microenvironment and enhanced immune response. Cellular immunology. 2011; 271(2):227-238. https://doi.org/10.1016/j.cellimm.2011.07.001

49. Chowdhury RR, Fitch RW, Ghosh SK. Efficacy of phytol-derived diterpenoid immunoadjuvants over alum in shaping the murine host's immune response to Staphylococcus aureus. Vaccine. 2013;31(8):1178-1186. https://doi.org/10.1016/j.vaccine.2012.12.069

50. Kiani M, Zamani Z, Khalighi A, Fatahi R, Byrne DH. Microsatellite analysis of Iranian Damask rose (Rosa damascena Mill.) germplasm. Plant Breeding. 2010; 129(5): 551-557. https://doi.org/10.1111/j.1439-0523.2009.01708.x

51. Schmitzer V, Veberic R, Osterc G, Stampar F. Color and phenolic content changes during flower development in groundcover rose. A Soci Horti Scie. 2010; 135(3): 195-202. https://doi.org/10.21273/JASHS.135.3.195

Page $12 / 15$ 
52. Tanaka Y, Sasaki N, Ohmiya A. Biosynthesis of plant pigments: Anthocyanin's, betalains and carotenoids. Plant J. 2008 54(4): $733-749$. https://doi.org/10.1111/j.1365-313X.2008.03447.x

53. Ackermann IE, Banthorpe DV, Fordham WD, Kinder JP, Poots I. $\beta$-Glucosides of aroma components from petals of Rosa species: Assay, occurrence, and biosynthetic implications. Plant Physiol. 1989; 134(5): 567-572. https://doi.org/10.1016/S0176-1617(89)80148-8

54. Winterhalter P, Sefton MA, Williams PJ. Two-dimensional GC-DCCC analysis of the glycoconjugates of monoterpenes, norisoprenoids, and shikimatederived metabolites from Riesling wine. J. of Agri and Food Chem. 1990; 38(4): 1041-1048. https://doi.org/10.1021/jf00094a028

55. Cherchi G, Deidda D, Gioannis BD, Marongiu B, Pompei R, Porcedda S. Extraction of Santolina insularis essential oil by supercritical carbon dioxide: Influence of some process parameters and biological activity. Flavour and Fragr J. 2001; 16(1): 35-43. https://doi.org/10.1002/10991026(200101/02)16:1\%3C35::AID-FFJ942\%3E3.0.CO;2-Y

56. Jamoussi B, Romdhane M, Abderraba A, Hassine BB, Gadri AE. Effect of harvest time on the yield and composition of Tunisian myrtle oils. Flavour and Fragr J. 2005; 20(3): 274-277. https://doi.org/10.1002/ffj.1453

57. Marotti M, Piccaglia R, Giovanelli E, Deans SG, Eaglesham E. Effects of planting time and mineral fertilization on peppermint (Mentha x piperita L.) essential oil composition and its biological activity. Flavour and Fragr J. 1994; 9(3): 125-129. https://doi.org/10.1002/ffj.2730090307

58. Croteau R, Gershenzon J. Genetic control of monoterpene biosynthesis in mints (Mentha: Lamiaceae). In: Genetic Engineering of Plant Secondary Metabolism. 1994. pp: 193-229. https://doi.org/10.1007/978-1-4615-2544-8_8

59. Kothari SK, Singh UB. The effect of row spacing and nitrogen fertilization on scotch spearmint (Mentha gracilis Sole). J of Esse Oil Rese. 1995; 7(3): 287297. https://doi.org/10.1080/10412905.1995.9698521

60. Farooq A, Khan MA, Ali A, Riaz A. Diversity of morphology and oil content of Rosa damascena landraces and related Rosa species from Pakistan. Pakistan Journal of Agri Sci. 2011; 48(3):177-183.

61. Baydar H, Schulz H, Krüger H, Erbas S, Kineci S. Influences of fermentation time, hydro-distillation time and fractions on essential oil composition of Damask Rose (Rosa damascena Mill.). J. of Essential Oil Bearing Plants. 2008; 11(3): 224-232. https://doi.org/10.1080/0972060X.2008.10643624

62. Kumar R, Sharma S, Kaundal M, Sood S, Agnihotri VK. Variation in essential oil content and composition of Damask Rose (Rosa damascena Mill) flowers by salt application under mid hills of the western Himalayas. J of Esse Oil Bearing Plants. 2016; 19(2): 297-306.

https://doi.org/10.1080/0972060X.2016.1153985

63. Mohamadi M, Mostafavi A, Shamspur T. Effect of storage on essential oil content and composition of Rosa damascena Mill. petals under different conditions. J of Essen Oil Bearing Plants. 2011; 14(4), 430-441. https://doi.org/10.1080/0972060X.2011.10643598

64. European Pharmacopoeia. European directorate for the quality of medicines and healthcare, Council of Europe. 8th edn. 2014. Vol. 1.

\section{Figures}

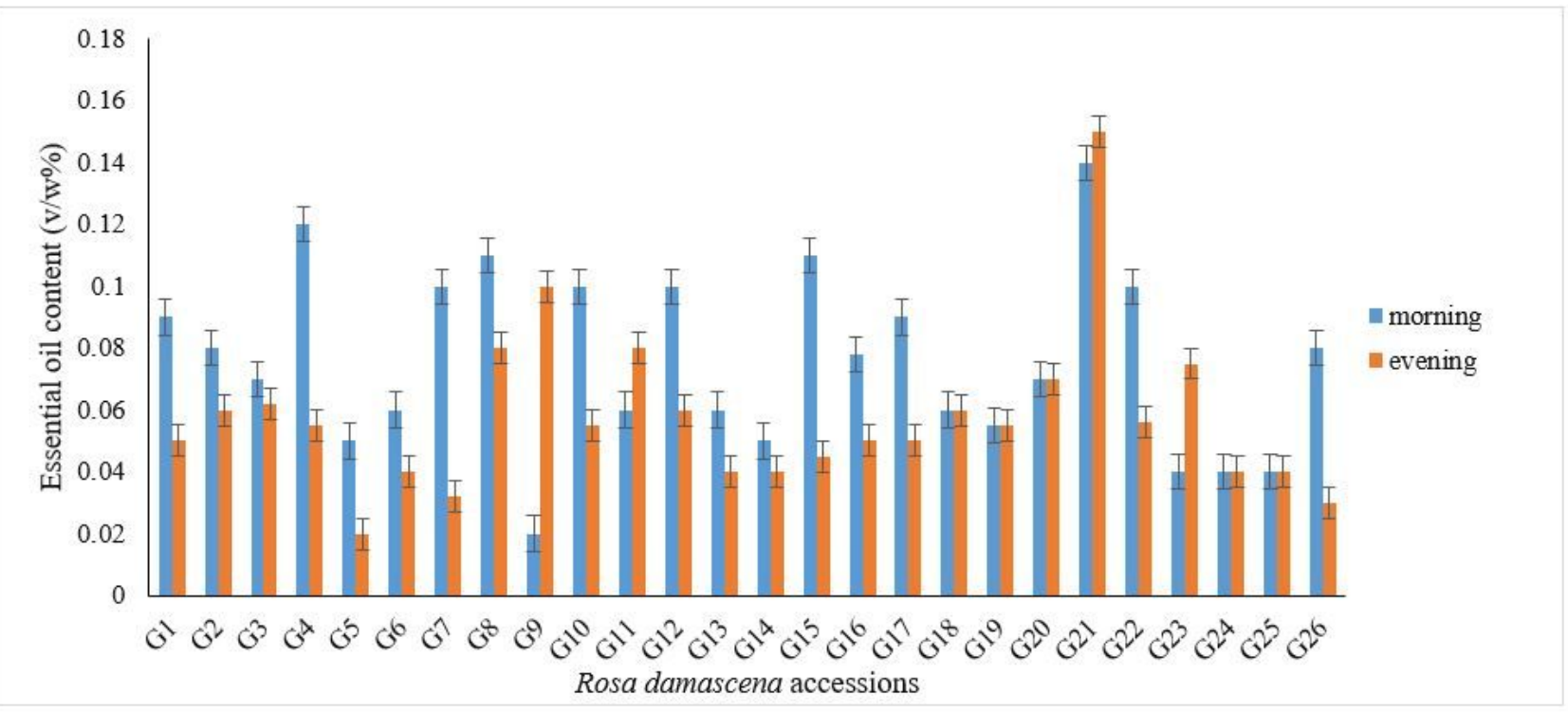

Figure 1

Essential oil content of fresh petals of Rosa damascena harvested in the morning and evening. 


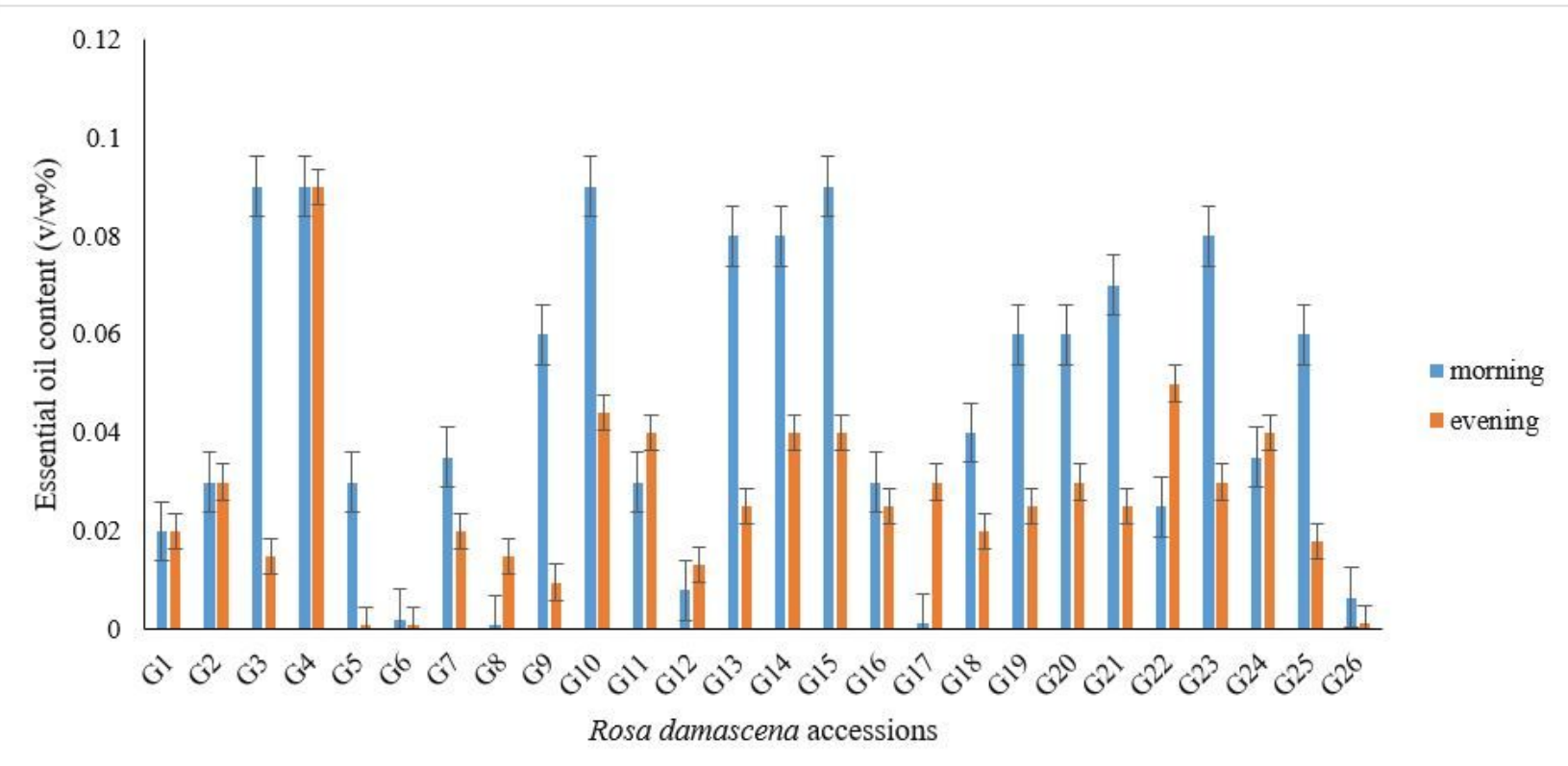

Figure 2

Essential oil content of dry petals of Rosa damascena harvested at morning and evening
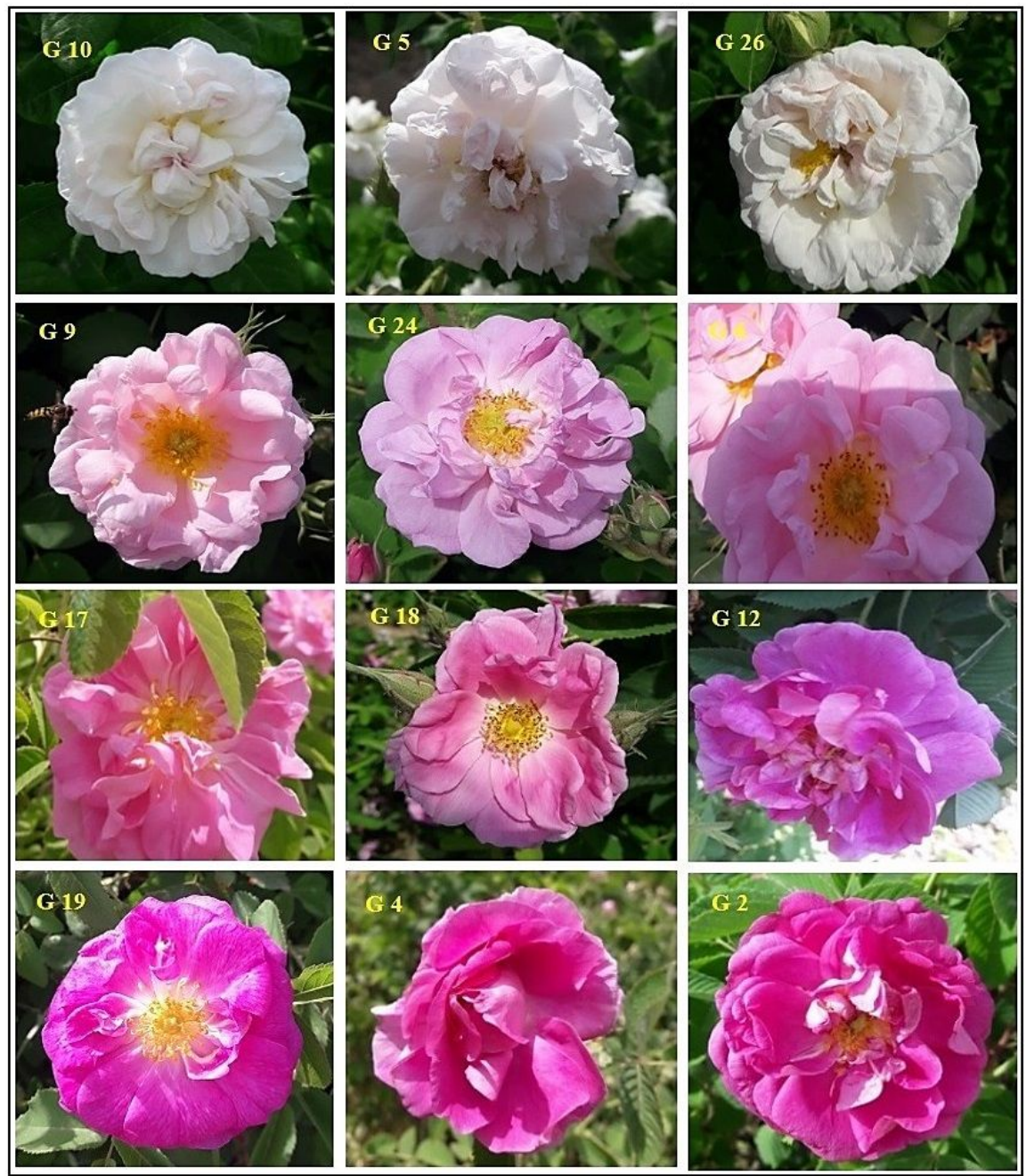

Figure 3 
Full blooming flowers of some Rosa damascena genotypes in the present study

Page 15/15 